\title{
Análise da Preferência do Pescador Artesanal do Município de Pentecoste (CE) por Programas de Peixamento ${ }^{1}$
}

\author{
Jonathas Viana Monteiro², Alana Kedylla Monteiro Norões ${ }^{3}$ e \\ Rogério César Pereira de Araújo ${ }^{4}$
}

\begin{abstract}
Resumo: A pesca continental tem papel importante para a subsistência e renda das famílias no meio rural na região Nordeste do Brasil. Visando aumentar a captura da pesca continental, o Departamento Nacional de Obras Contra as Secas (DNOCS) criou o programa de Peixamento em açudes públicos. Este estudo tem como objetivo revelar a preferência do pescador, que reside no município de Pentecoste (CE), pelo programa de peixamento com base em sua disposição a pagar por cenários de pescaria. Para isto, estimam-se as disposições a pagar (DAP) por atributos que influenciam a escolha do pescador por açude por meio do método de valoração ambiental, denominado Modelagem de Escolha. Para uma amostra de 100 pescadores, as disposições a pagar foram estimadas com base nos modelos logit condicional e misto. A DAP média do pescador por pescar em açude peixado foi cerca de R \$50,00/dia, o que representa o aumento no nível de bem-estar do pescador gerado a partir do programa de Peixamento do DNOCS. A DAP média do pescador para substituir a experiência corrente de pesca (status quo) por qualquer outro cenário proposto foi de $R \$ 89,00 /$ dia, o que revela as oportunidades de melhoria da pesca em açudes no semiárido da região Nordeste.
\end{abstract}

Palavras-chaves: modelagem de escolha, pesca artesanal, política pública.

Abstract: The continental fishing has an important role to the livelihood and income of the rural families of the Northeastern region in Brazil. Aiming to increase the catch level of the continental fishing, the National Department of Drought Works (DNOCS) has created the fish stocking program of the public dams. This research aims to reveal the fishers' preference that live in the municipality of Pentecoste (Ceará State) towards the fish stocking program having as basis their willingness to pay (WTP) for fishing scenarios. To do that, we estimate the willingness to pay for attributes which affect the fisher's choice for reservoir by the method of environmental valuation, called Choice Experiment. Based upon a sample of 100 fishers, the willingness to pay was estimated according to the conditional

1. Data de submissão: 4 de maio de 2017. Data de aceite: 26 de novembro de 2017.

2. Universidade Federal do Ceará, Fortaleza, Ceará, Brasil. E-mail: jonathas0801@ hotmail.com

3. Universidade Federal do Ceará, Fortaleza, Ceará, Brasil. E-mail: alanadylla@hotmail.com

4. Universidade Federal do Ceará, Fortaleza, Ceará, Brasil. E-mail: rcpa@ufc.br 
and mix logit models. The fisher's mean WTP to fish at a stocked dam was 50,00 BRL/day that shows the increased fisher's wellbeing due to the DNOCS's fishing stocking program. The fisher's mean WTP to substitute the current fishing experience (status quo) by any other proposed scenario was 89,00 BRL/day, revealing the opportunities of improving the fishing at dams of the semiarid Northeastern region.

Key-words: modeling choice, fisheries, public policy.

Classificação JEL: A13, C01, C12, C35, C54, D60, D62, Q00, R00.

DOI: http://dx.doi.org/10.1590/1234-56781806-94790560308

\section{Introdução}

No Brasil, a pesca artesanal é uma importante fonte de renda e proteína animal para muitas famílias, sendo esta relevante para a manutenção cultural das comunidades de pescadores. Em 2007, esta modalidade de pesca foi responsável por $47,1 \%$ de todo o pescado produzido no País (BRASIL, 2015).

Dentre as modalidades de pesca artesanal, a extrativista continental tem se destacado por sua importância socioeconômica na região Nordeste do Brasil. Esta atividade é tipicamente realizada por pescadores com baixo nível de escolaridade, sendo uma ocupação que é passada de geração para geração (SILVA, 2011). Desta forma, contribui de forma marcante para a segurança alimentar, geração de ocupação e renda e redução do êxodo rural.

A pesca continental é uma atividade tradicionalmente praticada nos açudes do Nordeste brasileiro. Estima-se que a produção média de peixe nos açudes do Nordeste seja da ordem de $120 \mathrm{~kg} / \mathrm{ha}$.ano, em grande parte originada da pesca artesanal. Apesar de sua importância, historicamente, sua gestão tem sido negligenciada e, como consequência, conflitos e ameaças diversas têm surgido, por exemplo: conflitos territoriais (por área de pesca); conflitos entre modalidades de pesca (profissional e esportiva); vulnerabilidade à degradação ambiental (SALES, 2001).

O Departamento Nacional de Obras Contra as Secas (DNOCS), diante do declínio do estoque de peixes nos açudes e da necessidade de aumentar a dispo- nibilidade de proteína animal para a população rural, tem conduzido o programa de peixamento dos açudes públicos sob seu gerenciamento. Um dos primeiros peixamentos ocorridos no Nordeste foi realizado pelo DNOCS, no dia 14 de agosto de 1933, no açude "Campos da Sementeira", município de Arcoverde, em Pernambuco (SILVA e PINHEIRO, 2013).

O peixamento é definido como a atividade realizada com a finalidade de povoamento, repovoamento e suplementação de coleções d'água, com larvas, pós-larvas, alevinos, juvenis e adultos de peixes, crustáceos, moluscos, mamíferos etc. A introdução de indivíduos nas populações (peixamento) é uma prática importante para o restabelecimento de estoques, principalmente onde esses estão escassos ou se aproximando do esgotamento (SILVA e PINHEIRO, 2013).

A produção e distribuição de peixes são realizadas com base na adoção de Normas Técnicas de Boas Práticas de Manejo e de determinações específicas implantadas pelo DNOCS. De acordo com estas normas, o alevino utilizado no peixamento deve estar, em média, com 45 dias de vida, o que lhe dá maiores chances de sobreviver em meio aos predadores. A escolha das espécies utilizadas para peixamento deve levar em consideração as características de rusticidade, reprodução, migração, valor comercial, regime alimentar, área de incidência, peso, comprimento, e estudos realizados em seus habitats naturais e cativeiro (SILVA, 2009).

Ao longo dos anos, o programa de peixamento tem sido descontinuado, principalmente em virtude da falta de recursos financeiros suficientes para manter 
o programa ou devido à ocorrência de secas prolongadas que reduzem a cota dos açudes a um nível crítico, o que torna inviável a estocagem de peixes. Apesar dessas dificuldades, desde a sua criação, em 1932, o DNOCS introduziu 42 espécies de outros países ou de outras bacias nacionais, das quais 14 tipos de peixe e um de camarão se adaptaram às condições climáticas da região e chegaram a estabelecer populações viáveis nos açudes (SILVA, 2009).

O programa de peixamento tem como pressuposto fundamental a relação entre a estocagem de peixes nos açudes e o aumento da captura de peixes, embora tal relação seja difícil de ser confirmada empiricamente. Por outro lado, o programa tem sido conduzido sem considerar explicitamente na sua formulação as preferências do pescador, particularmente quanto às espécies a serem introduzidas, a frequência e a quantidade de estocagem.

Além desses fatores, pressupõe-se que o benefício esperado da pescaria dependa do fato de o açude ter sido estocado ou não, as espécies-alvos, a quantidade esperada de captura e a forma de gerenciamento dos açudes. Portanto, entender melhor a preferência do pescador por esses atributos pode auxiliar na gestão dos açudes e, especificamente, na elaboração de programas de peixamento que maximizem a utilidade esperada pelo pescador.

Portanto, este estudo tem como foco a descrição do perfil da pesca e a aferição da preferência do pescador por experiência de pescaria em açudes no semiárido brasileiro. Para isto, cenários hipotéticos que retratam as experiências de pescaria são construídos e, com base nas escolhas de onde pescar feitas pelo pescador, as disposições a pagar do pescador pelos atributos e níveis dos cenários são estimadas por meio de modelos de regressão. Este método é denominado de Modelagem de Escolha (ou Experimento de Escolha) e tem como fundamento a abordagem de preferência declarada.

O estudo empírico sobre a preferência dos pescadores artesanais é feito no município de Pentecoste (CE). O principal corpo d'água localizado no município é o açude Pereira de Miranda, com capacidade de 395,63 milhões de metros cúbicos (COGERH, 2016). Em Pentecoste, também está o laboratório de aquicultura Rodolpho Von Ihering, que produz alevinos que são destinados à venda e ao programa de peixamento dos açudes.
Este artigo é composto, além da introdução, por mais quatro seções. Na segunda seção apresentam-se os aspectos teóricos da modelagem de escolha e algumas aplicações do método. Na terceira seção está a metodologia da pesquisa, em que se delimita a área de estudo, descreve o método de valoração e os procedimentos de amostragem e análise dos dados. Na quarta seção são mostrados os resultados do estudo, particularmente as análises de regressão e das medidas de bem-estar. Finalmente, na quinta seção foram destacadas as principais conclusões do estudo.

\section{Revisão de literatura}

O programa de peixamento é uma forma de fixar o homem no seu local de origem, contribuindo para o bem social, geração de renda e incremento na qualidade da alimentação das comunidades ribeirinhas, além da manutenção e reposição de estoques pesqueiros (SALES, 2001). Apesar disso, o programa de peixamento e seus impactos socioeconômicos têm sido pouco investigados no Brasil, destacando-se os trabalhos de Sales (2001), Silva e Pinheiro (2013) e Sales (2015).

Sales (2001), ao avaliar os peixamentos realizados em açudes do sertão pernambucano, verificou que o programa beneficiou 44 mil pessoas, incluindo $11 \mathrm{mil}$ pescadores ( 10 por açude) e familiares ( 3 por pescador) que participavam das fainas de pesca, conserto e fabricação de canoas e apetrechos de pesca, e comercialização do pescado.

Silva e Pinheiro (2013), avaliando a sustentabilidade do programa de peixamento no município de Canindé (CE), estimaram os índices de sustentabilidade das dimensões social, ambiental, econômica e institucional do programa. Considerando-se que os índices variaram no intervalo [0,1], o programa apresentou nível baixo de sustentabilidade econômica $(0,343)$ e mediano de sustentabilidade institucional $(0,652)$.

Sales (2015) verificou que, no município de Pentecoste (CE), no período de 2005 a 2014, o DNOCS foi decisivo quanto à manutenção da produtividade pesqueira dos reservatórios beneficiados pelo programa de peixamento, apresentando-se como uma ferramenta essencial para fomentar a aquicultura familiar e empresarial, subsidiando a manutenção dos 
estoques e garantindo trabalho, renda e segurança alimentar. Neste período, pôde-se observar que, dentre os estados beneficiados, o que obteve o maior número de peixamentos foi o Ceará, seguido pelo Maranhão e Piauí.

No Brasil, inexistem trabalhos que tratem especificamente da valoração do peixamento de reservatórios. Porém, Rodrigues (2010) utilizou o mesmo método de valoração empregado neste artigo para estimar os valores indiretos associados ao recurso pesqueiro da região do lago de Manacapuru em Manaus (AM) para duas modalidades de pesca - comercial e subsistência. Utilizando o mesmo método, Araújo e Coutinho (2016) investigaram a preferência dos irrigantes com relação aos métodos de cobrança de água bruta para irrigação no perímetro irrigado de Tabuleiro de Russas, localizado no estado do Ceará.

A modelagem de escolha foi aplicada para investigar a preferência dos pescadores pelos atributos da pesca e do peixamento de reservatórios na Alemanha e Estados Unidos.

Ditton (2004) utilizou a abordagem de escolha de preferência declarada para descrever as preferências dos pescadores do estado do Texas (EUA) pelos regulamentos e aspectos da pesca continental. Sete atributos foram utilizados para construir os cenários de pescaria: capacidade máxima da rede de espera, tamanho mínimo e máximo de peixe capturado, capacidade em reter peixes grandes, tamanho médio do peixe capturado, probabilidade de captura e custo de viagem.

Utilizando modelos logit condicionais, Ditton verificou que $23 \%$ dos pescadores prefeririam capturar peixes maiores em pequena quantidade, ao invés de um grande número de peixes pequenos, enquanto $51 \%$ prefeririam capturar um maior número de peixes, ao invés de peixes grandes em menor número. Foi visto que $26 \%$ dos pescadores apresentaram preferência pela existência de regulamentos quanto ao controle de captura, ao invés de ser livre a quantidade capturada por cada pescador. A maioria dos pescadores amostrados $(77,3 \%)$ preferiu trocar o cenário corrente por qualquer cenário alternativo apresentado nos conjuntos de escolha.

Arlinghaus et al. (2014) utilizaram a modelagem de escolha para aferir a preferência dos pescadores pelo programa de peixamento e quanto aos resultados das capturas, na Alemanha. Utilizando uma amostra de 1.335 pescadores, verificaram que a captura de peixes nativos influenciava negativamente, de forma significativa, a utilidade dos pescadores. Já a frequência de estocagem e experiência dos pescadores proporcionaram ganhos de utilidade para o pescador continental. Foi observado também que o pescador tem maior preferência em pescar em reservatórios que são peixados, pois, com isso, ele espera elevar o nível de sua captura. Além disso, eles apresentaram maior preferência em pescar espécies exóticas, ao invés de capturar peixes nativos e preferiam sempre maior captura diária, ao invés da captura referente à sua situação corrente.

Este artigo pretende expandir o conhecimento sobre as preferências por experiências de pesca no Brasil e estimar o valor dos atributos da pescaria em açudes públicos realizada por pescadores artesanais no estado do Ceará e, particularmente, colocar em evidência a importância do peixamento dos açudes para o bem-estar do pescador.

\section{Metodologia}

\section{1. Área de estudo}

A pesquisa foi realizada com os pescadores artesanais que residiam no município de Pentecoste (CE) e realizam a pesca no açude Pereira de Miranda e em outros açudes no interior do Ceará. Pentecoste, também conhecida como "Terra do Peixe", está localizado na mesorregião norte do estado do Ceará. Este município ocupa área de $1.378,30 \mathrm{~km}^{2}$ e a população, em 2017, era de 37.077 habitantes (IBGE, 2017).

A economia deste município está baseada na pesca continental de subsistência e na agricultura de subsistência das culturas de milho, feijão e mandioca, além de banana e coco em áreas irrigadas, próximas à faixa do rio Curu perenizado e do açude Pereira de Miranda (CEARÁ, 2015).

O açude Pereira de Miranda, localizado no município de Pentecoste, foi construído em 1957 e é conhecido popularmente como "Açude Pentecoste". Este açude tem capacidade de armazenamento de $395.638 .000 \mathrm{~m}^{3}$ (CEARÁ, 2015); porém, em 2016, seu volume correspondia a apenas $1,05 \%$ de sua capacidade em virtude do período de seca prolongado (COGERH, 2016). Além da pesca artesanal, as águas deste reservatório são utilizadas para a piscicultura superintensiva em tanques-rede (COGERH, 2016). 


\subsection{Modelagem de escolha}

A modelagem de escolha foi desenvolvida por psicólogos matemáticos em 1960, com o objetivo de transformar escolhas subjetivas em parâmetros calculados. Este método possibilita valorar as características ou atributos de um bem e de alterações nestas características de forma mais direta, em vez de valorar o bem como um todo (BATEMAN et al., 2002).

Essa modelagem permite que os indivíduos identifiquem a utilidade das características dos bens, possibilitando uma estimação multidimensional de vários atributos de um bem. As vantagens desse método estão associadas ao fato de o respondente fazer escolhas ao invés de manifestar explicitamente valores monetários e permitir fazer uso mais eficiente da amostra, visto que um número maior de respostas é obtido de cada indivíduo (BATEMAN et al., 2002).

Uma função de utilidade é especificada em termos dos atributos, aos quais são atribuídos pesos. Esses pesos, também chamados de valor parte (part worth), expressam a importância relativa de cada atributo conforme as escolhas do indivíduo. Na modelagem de escolha, um dos atributos é o preço ou custo do bem sendo valorado, o qual permite estimar a disposição a pagar ou a aceitar pelos custos/benefícios ambientais (BATEMAN et al., 2002).

Nas subseções seguintes, a aplicação do método de modelagem ao contexto deste estudo é descrita.

A primeira etapa do método consiste em caracterizar o problema de decisão que irá orientar a valoração. O problema de decisão está relacionado com o momento em que o pescador engaja no processo de avaliação das características de um conjunto de alternativas de pescaria que dispõe (conjunto de escolha) e que resulta na escolha do local de pesca que maximize sua utilidade esperada da pescaria. Para isto, as pescarias alternativas devem ser descritas por meio de atributos e níveis.

A segunda etapa do método consiste na seleção dos atributos da pesca continental no semiárido e seus níveis. Para isto, entrevistas foram realizadas com técnicos do DNOCS, que têm contato direto com pescadores, e com o chefe da colônia de pescadores de Pentecoste. Depois de criteriosa avaliação dos atributos propostos, foram descritos os cenários de pescaria com base na combinação dos níveis de quatro atributos, a saber: (i) espécies-alvo da pesca continental no semiá- rido; (ii) programa de peixamento; (iii) tempo de pesca e (iv) produtividade da pesca continental.

O primeiro atributo, espécies-alvo da pesca continental no semiárido, descreve as espécies que o pescador geralmente captura nos açudes ou reservatórios, dentre elas aquelas que são estocadas pelo programa de peixamento do DNOCS. Tipicamente, o pescador tem preferência por determinada espécie de peixe, que pode estar associada aos seus hábitos alimentares ou ao benefício financeiro.

Nesse contexto, levando em consideração as espécies frequentemente capturadas em açudes, foram propostos três grupos de espécies alvo: tilápia e peixes amazônicos (tambaqui, pirarucu, pacu, curimatã, paqui, tambacu etc.). Desta forma, os níveis considerados para o experimento de escolha são: (i) qualquer espécie; (ii) tilápia como espécie de maior interesse em capturar; (iii) peixes amazônicos como espécie de maior interesse em capturar.

O segundo atributo expressa o caráter do açude quanto ao peixamento, ou seja, se estocado ou não pelo programa de peixamento do DNOCS. Este atributo tem o intuito de avaliar a preferência do pescador quanto ao programa de peixamento realizado pelo DNOCS e, assim, verificar se o pescador associa ganhos de utilidade quando realiza pescaria em açudes peixados. Desta forma, a reposta do pescador em relação ao programa pode revelar a aceitação ou não do programa de peixamento por parte do pescador. Portanto, o atributo que expressa a situação do açude quanto ao peixamento será descrito por dois níveis: (i) "com" peixamento e; (ii) "sem" peixamento.

O terceiro atributo, tempo de pescaria, expressa o esforço de pesca do pescador, que é o tempo que este passa pescando diariamente. O tempo de pesca será utilizado como proxy para o custo de oportunidade que o pescador encara em sua atividade de pesca em face a outra atividade que gera renda também em sua localidade ${ }^{5}$.

A maioria dos pescadores que atua no semiárido trabalha também no setor agrícola como diarista ou pratica outra atividade para aumentar a renda mensal familiar. Em razão dos incentivos serem poucos para

5. O custo de oportunidade é um termo usado em economia para indicar o custo de algo, em termos de uma oportunidade renunciada, que foi definido por Buchanan (1987) como uma expressão da relação básica entre escassez e escolha. 
o pescador permanecer na atividade, pretende-se aferir - caso o cenário contivesse os aspectos relacionados à pesca continental que favorecessem a atividade pesqueira nos açudes do semiárido - se ele dedicaria um tempo maior nessa atividade, buscando tê-la como sua principal fonte de renda, ao invés de realizá-la somente como um complemento de renda, ou como forma de subsistência. ${ }^{6}$

Os valores escolhidos para representar os níveis do tempo de pesca foram estabelecidos por meio de entrevistas com técnicos do DNOCS que realizavam coleta de informações da atividade pesqueira diretamente com os pescadores que atuavam e residiam no município de Pentecoste. Desta forma, foram selecionados três níveis: (i) 2 horas de pesca (tempo mínimo); (ii) 4 horas de pesca (tempo médio) e (iii) 6 horas de pesca (tempo máximo).

$\mathrm{O}$ quarto atributo, produtividade da pesca continental, é definido em termos da quantidade capturada em um dia de pescaria, medido em $\mathrm{kg} /$ dia. Este tem o objetivo de refletir o rendimento médio do pescador na pescaria. Os valores de captura foram obtidos por meio de entrevistas realizadas com técnicos do DNOCS, sendo baseados em informações fornecidas pelos pescadores quanto à captura diária obtida nos açudes do Nordeste.

Portanto, nesse contexto, três níveis de produtividade da pesca continental foram considerados para o experimento de escolha: (i) captura mínima de $3 \mathrm{~kg} / \mathrm{dia}$; (ii) captura média de $5 \mathrm{~kg} /$ dia e (iii) captura máxima de $8 \mathrm{~kg} /$ dia.

A terceira etapa do método consiste em desenvolver o desenho experimental que consiste da construção dos diferentes cenários alternativos, gerados a partir da combinação dos níveis dos atributos. O desenho experimental permite escolher conjuntos de alternativas a partir do total de alternativas possíveis, de forma estatisticamente eficiente, para serem incluídas no questionário (BATEMAN et al., 2002).

6. Segundo o IBGE (2010), o valor do rendimento nominal médio mensal de todos os trabalhos das pessoas do município de Pentecoste, de 10 anos de idade ou mais, em 2010 era de R\$ 832,04/mês. Considerando-se 20 dias de trabalhados, o valor diário do rendimento nominal era de $\mathrm{R} \$ 41,60 /$ dia. Com base nesse valor - corrigido por meio do Índice Geral de Preços (IGP-M), que é um indicador de movimento dos preços calculado mensalmente pela Fundação Getúlio Vargas (FGV), para 2016 - será elaborada a variável "custo de oportunidade" (COPORT), levando-se em consideração o tempo de pesca.
Nesta pesquisa, utilizou-se o planejamento fatorial fracionário dos efeitos principais, que tem a propriedade da ortogonalidade. Esta propriedade garante que cada atributo incluído na alternativa não esteja correlacionado com qualquer dos outros atributos. Somado a isto, utiliza-se o delineamento dos efeitos principais que assume ser a utilidade de cada alternativa dependente direta de cada atributo, ou seja, desconsideram-se os efeitos que possam se originar de interações entre atributos (BATEMAN et al., 2002).

A combinação fatorial completa dos atributos que descreve a pescaria é calculada pela seguinte expressão: $n^{\mathrm{a}} \cdot m^{\mathrm{b}}$; em que a base de cada potência é o número de níveis de cada atributo $(n=3$ e $m=2)$ e o expoente é o número de atributos que tem o mesmo número de níveis ( $a=3$ e $b=1$ ). Assim, o total de combinações possíveis dos níveis dos atributos é igual a $54\left[=3^{3} \cdot 2^{1}\right]$. O pacote estatístico SPSS Statistics Base 20 foi utilizado para definir o desenho mínimo eficiente do planejamento fatorial fracionário dos efeitos principais. Do total de alternativas possíveis, foram selecionados nove cenários para o desenho experimental.

A quarta etapa consiste na construção dos conjuntos de escolha a serem avaliados pelos respondentes. Nesta pesquisa, seguindo a recomendação de Pearce (2006), um conjunto de escolha é definido por três opções: dois cenários alternativos e o cenário corrente $(S Q)$. Esta última opção representa a opção de desistência (ou opt-out), ou seja, quando o pescador prefere a situação corrente a qualquer um dos cenários propostos.

O número de combinações possíveis não ordenadas dos cenários totalizou 36 pares, obtida pela fórmula: $c \cdot(c-1) / 2$, em que $c$ é o número de cenários $(c=9)$. Os 36 conjuntos de escolha foram divididos em seis subgrupos, cada um formado por seis conjuntos de escolha. A distribuição dos conjuntos de escolha em subgrupos permitiu reduzir o tamanho da amostra e aumentar a eficiência da análise das preferências.

A situação corrente da pescaria é a linha de referência da análise e é chamado de Pescaria Atual ou Status Quo (SQ), sendo que existe uma situação corrente para cada pescador. Em cada conjunto de escolha, o pescador compara a sua experiência da pescaria corrente com os cenários alternativos propostos e escolhe aquela que maximiza sua utilidade esperada.

O Quadro 1 mostra a composição dos cenários conforme o desenho experimental mínimo eficiente. 
Quadro 1. Desenho ortogonal de efeito principal gerado a partir do software estatístico IBM SPSS Statistics Base 20, em 2016

\begin{tabular}{|c|c|c|c|c|}
\hline Cenários & $\begin{array}{c}\text { Espécies alvo } \\
\text { de Peixes }\end{array}$ & Peixamento & $\begin{array}{c}\text { Produtividade } \\
\text { (kg/dia) }\end{array}$ & $\begin{array}{c}\text { Tempo de pesca } \\
\text { (hora/dia) }\end{array}$ \\
\hline C1 & Amazônicos & Com & 8 & 4 \\
\hline C2 & Qualquer espécie & Sem & 8 & 6 \\
\hline C3 & Tilápia & Com & 8 & 2 \\
\hline C4 & Tilápia & Com & 5 & 2 \\
\hline C5 & Qualquer espécie & Com & 3 & 6 \\
\hline C6 & Amazônicos & Com & 3 & 4 \\
\hline C7 & Qualquer espécie & Com & 3 & 2 \\
\hline C8 & Amazônicos & Sem & 5 & 4 \\
\hline C9 & Tilápia & Sem & 3 & \\
\hline
\end{tabular}

Fonte: Elaborado pelos autores.

Em face da utilização do esquema de comparações foi necessário construir combinações de conjuntos de escolha de forma que o arranjo apresentado dos cenários pudesse facilitar o experimento para o respondente. De outra mão, o cotejo de cenários com elementos completamente diferentes entre si dificultaria a avaliação sobre qual atributo teria maior peso na escolha do respondente.

Sendo assim, inicialmente, para a composição dos conjuntos de escolha, foram selecionados cenários que apresentassem o maior número de elementos em comum com o Cenário Base. Em seguida, foram agru- pados cenários com mais elementos comuns entre si. Cada cenário foi representado visualmente utilizando imagens que retratavam os atributos, seguindo a sugestão de Coutinho (2015). As imagens foram selecionadas e editadas a partir de vetores extraídos do site br.freepik. com, conforme o Quadro 2.

Posteriormente, as imagens foram agrupadas para a composição dos cenários gerados a partir do desenho ortogonal. O cenário da situação corrente (status quo) é representado pelo termo "PESCARIA ATUAL". A Figura 1 apresenta um exemplo de cenário e o status quo da pescaria.

Quadro 2. Imagens dos níveis dos atributos utilizadas no experimento de escolha

\begin{tabular}{|c|c|c|c|}
\hline Nível do Atributo & Imagem & Nível do Atributo & Imagem \\
\hline Qualquer espécie, sem espécie-alvo específica & & 2 horas de pesca (tempo mínimo) & \\
\hline Tilápia como espécie-alvo & & 4 horas de pesca (tempo médio) & \\
\hline Peixes amazônicos como espécie-alvo & & 6 horas de pesca (tempo máximo) & \\
\hline Com peixamento & & Produtividade de $3 \mathrm{~kg} /$ dia & \\
\hline Sem peixamento & & Produtividade de $5 \mathrm{~kg} / \mathrm{dia}$ & \\
\hline & & Produtividade 8 kg/dia & \\
\hline
\end{tabular}

Fonte: Elaborado pelos autores. 
Figura 1. Exemplo de um conjunto de escolha (C1) e o status quo da pescaria

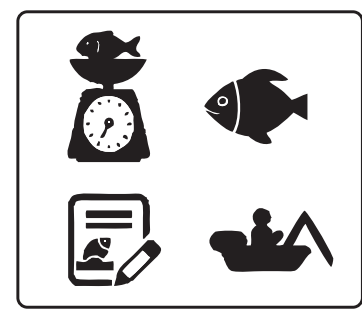

$\mathrm{C} 1$

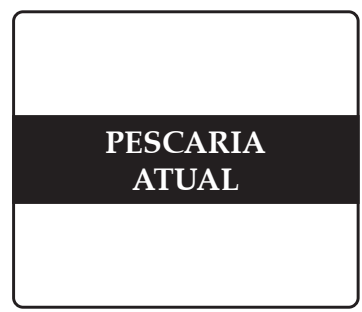

Status quo

Fonte: Elaborado pelos autores.

\subsection{Modelo econométrico}

O modelo de experimento de escolha assume que o pescador tenha preferência formada pelos atributos e níveis que descrevem a pesca continental, que possivelmente pode realizar. Neste contexto, um pescador - diante de um número fixo de alternativas $(j=1, \ldots, J)$ de experiências de pesca continental, cada uma delas descrita por um conjunto de atributos $(k=1, \ldots, K)$, dentre os quais inclui-se o açude ter sido peixado - irá escolher a opção $j$ se o nível de utilidade desta opção for maior do que o nível de utilidade oferecido por qualquer uma das demais alternativas.

A função de utilidade indireta $U_{i j}$ de um indivíduo $i$ que escolhe a alternativa $j$, a partir de um conjunto de escolha $C$, pode ser decomposta em uma parte determinística e observável $\left(V_{i j}\right)$, e outra estocástica $\left(\varepsilon_{i j}\right)$, não observável. Tipicamente, a parte determinística é especificada como a combinação linear das variáveis explicativas $\left(X_{i j}\right)$ com parâmetros $(\beta)$, como mostra a equação a seguir:

$$
U_{i j}=V_{i j}+\varepsilon_{i j}=\beta X_{i j}+\varepsilon_{i j}
$$

em que: $X_{i j}$ é o vetor das variáveis que descrevem as alternativas de escolha e as características socioeconômicas dos indivíduos.

Se o indivíduo selecionar a alternativa que maximize sua utilidade, então a probabilidade de o indivíduo $i$ escolher a alternativa $j$ ao invés de $h$, ambas pertencentes ao conjunto de escolha $C$, é dada pela seguinte expressão:

$$
\begin{aligned}
& \operatorname{Pr}(j \mid C)=\operatorname{Pr}\left(\beta X_{i j}+\varepsilon_{i j}>\beta X_{i h}+\varepsilon_{i h}\right)= \\
& \operatorname{Pr}\left(\varepsilon_{i j}-\varepsilon_{i h}>\beta X_{i h}-\beta X_{i j}\right)
\end{aligned}
$$

Assumindo que os termos do erro seguem a distribuição Gumbel, obtém-se o modelo logit condicional (BELL, SHAH e WARD, 2014), cuja probabilidade de escolha da alternativa $j$ feita pelo indivíduo $i$ é dada pela seguinte equação:

$$
\operatorname{Pr}\left(j \mid X_{i j,} \beta\right)=\frac{\exp \left(\beta X_{i j}\right)}{\sum_{j} \exp \left(\beta X_{i j}\right)}
$$

O modelo logit misto relaxa a suposição de alternativas independentes e irrelevantes que embasam o modelo logit condicional, permitindo que os parâmetros sejam distribuídos aleatoriamente na população. Desta forma, as preferências variam aleatoriamente entre os indivíduos, estando as escolhas condicionadas à especificação da distribuição dos coeficientes (McFADDEN e TRAIN, 2000; RIGBY, ALCON e BURTON, 2010), o que permite capturar a heterogeneidade das preferências.

Definindo a distribuição dos parâmetros $\beta$ pelo vetor de parâmetros $\varphi$ (tipicamente, a média e variância da distribuição), a probabilidade do indivíduo $i$ escolher a alternativa $j\left(P_{i j}^{\prime}\right)$, a partir de um conjunto de escolha $C$, é dada pela seguinte expressão (RIGBY, ALCON e BURTON, 2010).

$$
P_{i j}^{\prime}=\int \exp \left(\beta_{i} X_{i j}\right) / \sum_{i} \exp \left(\beta_{i} X_{i j}\right) f(\beta \mid \varphi) d \beta
$$

em que: $\beta_{i}$ é um vetor de parâmetros de preferências individuais; $f(\beta \mid \varphi)$ é a função de densidade de probabilidade para $\beta$ definido sobre um vetor de parâmetro $\varphi$. A matriz $\varphi$ define os parâmetros que caracterizam a distribuição dos parâmetros aleatórios (e.g., normal, log-normal, triangular etc.) definida pelo pesquisador. 
Os coeficientes de todos os atributos, variam de acordo com a distribuição normal e não correlacionados entre si (BELL, SHAH e WARD, 2014).

Neste estudo, segundo Barton e Bergland (2010), calcula-se a disposição a pagar (excedente de compensação) por uma mudança (melhoria) não marginal no conjunto de atributos da pesca continental conforme a seguinte equação:

$D A P=\beta_{y}^{-1} \ln \left[\frac{\sum_{j} \exp \left(V_{j}^{1}\right)}{\sum_{j} \exp \left(V_{j}^{0}\right)}\right]=$

$\beta_{y}^{-1}\left[\ln \sum_{j} \exp \left(V_{j}^{1}\right)-\ln \sum_{j} \exp \left(V_{j}^{0}\right)\right]$ com $j \in C$

em que: $V_{j}^{0}$ é a utilidade inicial da opção $j ; V_{j}^{1}$ é a utilidade final da opção $j ; \beta_{y}$ é a utilidade marginal da renda que corresponde ao coeficiente do atributo de custo e; $C$ é o conjunto de escolha apresentado ao indivíduo. As variações em $V_{j}^{0}$ e $V_{j}^{1}$ podem surgir a partir de mudanças nos atributos das alternativas ou exclusão (ou inclusão) de alternativas.

O coeficiente de um atributo $\left(\beta_{k}\right)$ expressa a preferência de um indivíduo por este atributo, podendo ser interpretado como a utilidade marginal. Portanto, a disposição a pagar $(D A P)$ por um atributo é expressa pela taxa marginal de substituição deste atributo por dinheiro (BELL, SHAH e WARD, 2014). Assumindo que a parte determinística da utilidade é linear em seus parâmetros, a DAP para qualquer um dos atributos $(k)$, também denominada de custo implícito, é calculada pela seguinte expressão para os modelos logit, condicional (Equação 6) e misto (Equação 7):

$$
\begin{aligned}
& D A P_{k}=-\beta_{k} / \beta_{y} \\
& E\left(D A P_{k}\right)=-E\left(\beta_{k}\right) / \beta_{y}
\end{aligned}
$$

O modelo empírico empregado nesta pesquisa corresponde a seguinte equação:

$$
\begin{aligned}
& U_{i j}=\beta_{0} A_{S C} C_{i j}+\beta_{1} \text { CAPTURA }_{i j}+ \\
& \beta_{2} \text { TILÁPIA } \\
& \beta_{4} \text { COPORT } \\
& \text { COPA }_{i j}+\beta_{5} \text { INTERACÇAO }_{i j}+\ldots+ \\
& \beta_{5+N} I N T E R A C ̧ A O_{i j}+\varepsilon_{i j}
\end{aligned}
$$

Na equação (8), o termo $U_{i j}$ representa a utilidade do pescador continental $i$ que escolhe a alternativa $j$, a partir de um conjunto de escolha. As variáveis explicativas do modelo também estarão relacionadas ao pescador $i$ e sua alternativa escolhida $j$, sendo elas: ASC, o indicador dos cenários propostos que não o status quo;
CAPTURA, a quantidade capturada de peixe; TILÁPIA, pescar a tilápia, ao invés de qualquer outra espécie; PXAMA, pescar peixes amazônicos, ao invés de qualquer outra espécie; COPORT, o custo de oportunidade do pescador, baseado no tempo de pesca; INTERAÇAO, uma variável que relaciona uma característica socioeconômica do pescador e um nível de atributo da pescaria; e $\varepsilon_{i j}$ o componente estocástico da equação.

Para a análise das preferências declaradas dos pescadores do município de Pentecoste, foram estimados quatro modelos logísticos: modelo logit condicional (MLC), simples e expandido (MLC 1 e 2) e modelo logit misto (MLM), simples e expandido (MLM 1 e 2). Os modelos logit simples, tanto o condicional como o misto (MLC 1 e MLM 1), consideram apenas os efeitos principais dos atributos, enquanto os modelos logit expandidos (MLC 2 e MLM 2) incluem ambos, tanto os atributos principais da pesca continental, quanto os termos de interação entre variáveis. Para estes modelos, a equação (8) será tomada como base para o processo de modelagem dos dados.

\subsection{Coleta de dados}

O universo da pesquisa é formado pelos pescadores de água doce que residem em Pentecoste. Pelo fato de não dispor da lista dos pescadores residentes no município, a amostragem empregada foi não probabilística e por conveniência.

Estima-se que existam 854 pescadores em Pentecoste. Este valor foi calculado com base no valor total do seguro defeso pago aos pescadores (R\$ 2.019.060), segundo dados do Ministério da Transparência, Fiscalização e Controladoria-Geral da União (BRASIL, 2015), número de meses do período de defeso (três meses) e valor do salário mínimo vigente ( $\mathrm{R} \$ 788,00)$ em 2015. O número estimado de pescadores foi de 854 pescadores $[=2.019 .060 /(3) \cdot(788)]$.

Dadas as limitações financeiras para a coleta de uma amostra representativa, a amostra foi determinada em função do número mínimo de observações necessárias para viabilizar a análise de regressão. Para isto, foram entrevistados 100 pescadores que correspondem aproximadamente a $12 \%$ do total estimado de pescadores e um total de 600 escolhas de cenários do experimento.

Um questionário semiestruturado foi elaborado com o objetivo de coletar dados sobre os aspectos 
demográficos e socioeconômicos do pescador, suas experiências de pesca e preferências por cenários hipotéticos de pescaria. O questionário é formado por cinco partes: (1) identificação do questionário e do respondente; (2) caracterização da pesca (artes de pesca, embarcações, espécies capturadas, tempo de pescaria etc.); (3) experimento de escolha (explicação da tarefa, conjuntos de escolha e questões follow up) e (4) caracterização demográfica e socioeconômica do pescador (idade, sexo, estado civil, tamanho da família, renda, educação etc.).

Os dados foram coletados em outubro de 2016. A tabulação e tratamento dos dados foram feitos utilizando uma planilha eletrônica do Excel, enquanto a análise estatística e econométrica foram feitas utilizando o pacote estatístico STATA 13.

\section{Resultados e discussão}

\subsection{Análise do perfil do pescador e da pesca}

Do total de 100 pescadores amostrados, a maioria deles era do sexo masculino, que representaram $88 \%$ da amostra. As mulheres pescadoras tiveram uma participação não desprezível na amostra de $12 \%$. Conforme a Tabela 1, a idade média dos pescadores é de aproximadamente 43 anos, variando no intervalo entre 18 e 80 anos. A média de tempo do pescador na atividade foi de 32,70 anos, variando entre 4 e 70 anos. O pescador típico tinha, em média, três filhos.

Sales (2001) encontrou resultados semelhantes ao estudar os pescadores no sertão pernambucano, em que a maioria dos pescadores era do sexo masculino, com idade média de aproximadamente 40 anos e com três filhos, em média.

Em relação ao nível de escolaridade, a maioria dos pescadores era analfabeto (35\%) ou possuía Ensino Fundamental incompleto (52\%), perfazendo um total de $87 \%$ da amostra (Tabela 2). Segundo os próprios res- pondentes, entre os pescadores existe uma alta taxa de evasão escolar que se atribui à necessidade de trabalhar já em idade jovem para ajudar na formação da renda familiar. Silva e Pinheiro (2013) também observaram baixo nível de escolaridade entre os pescadores de Canindé (CE).

Para 90\% dos pescadores, a maior parcela de sua renda familiar é proveniente do produto da pescaria que corresponde a mais de $80 \%$ (Tabela 2). Rodrigues (2010) também verificou que a pesca contribui para quase a totalidade da renda familiar mensal dos pescadores manauaras.

$\mathrm{Na}$ amostra, apenas uma pequena parte dos pescadores exercia outra atividade como fonte de renda, além da pesca, por exemplo: agropecuária (3\%), comércio $(2 \%)$, transferência governamental $(6 \%)$ e outras atividades (5\%). Silva e Pinheiro (2013) observou um percentual maior de pescadores $(87 \%)$, em Canindé, exercendo outras atividades como fonte de renda.

A renda do pescador, para $92 \%$ da amostra, variou no intervalo entre $R \$ 441,00$ e $R \$ 1.320,00$, sendo que $52 \%$ deles ganhavam menos do que $\mathrm{R} \$ 880,00$. A média estimada da renda foi de $R \$ 893,70$, que é ligeiramente maior do que o salário mínimo vigente em 2016, de $\mathrm{R} \$ 880,00$, e uma diária no valor de $\mathrm{R} \$ 29,33$.

Segundo os próprios respondentes, em 2016, a renda do pescador declinou consideravelmente devido à baixa precipitação pluviométrica e à descontinuidade do programa de peixamento nos açudes. Em períodos normais de chuvas e regularidade do peixamento, a média de renda do pescador pode ser até quatro vezes maior do que observada em 2016.

As variáveis de captura, apetrechos de pesca e comercialização do pescado são utilizadas para caracterizar a pesca no município de Pentecoste. O preço médio de venda do peixe foi de aproximadamente $\mathrm{R} \$ 3,30 / \mathrm{kg}$, podendo apresentar uma variação para mais ou para menos em torno de $1,30 \mathrm{Real} / \mathrm{kg}$, sendo que o menor valor de venda encontrado foi de $R \$ 2,00$ / $\mathrm{kg}$ e o maior, de $\mathrm{R} \$ 7,00 / \mathrm{kg}$ (Tabela 3). Em média, o pes-

Tabela 1. Estatística descritiva dos indicadores socioeconômicas dos pescadores do município de Pentecoste, em 2016

\begin{tabular}{lcccc}
\hline \multicolumn{1}{c}{ Indicadores } & Mín. & Máx. & Média & DP \\
\hline Tempo de pescaria & 4 & 70 & 32,7 & 12,821 \\
Idade & 18 & 80 & 43,29 & 12,99 \\
Número de filhos & 0 & 16 & 3,26 & 2,44 \\
\hline
\end{tabular}

Fonte: Elaborado pelo autor. 
Tabela 2. Análise dos fatores socioeconômicos dos pescadores do município de Pentecoste, em 2016

\begin{tabular}{|c|c|}
\hline Fatores & Percentual (\%) \\
\hline \multicolumn{2}{|l|}{ Escolaridade } \\
\hline Nem lê nem escreve & 35 \\
\hline Ens. Fundamental incompleto & 52 \\
\hline Ens. Fundamental completo & 5 \\
\hline Ens. Médio incompleto & 6 \\
\hline Ens. Médio completo & 2 \\
\hline Ens. Superior & 0 \\
\hline \multicolumn{2}{|l|}{ Proporção de contribuição da pesca na renda familiar } \\
\hline Menos de $40 \%$ & 0 \\
\hline 41 a $60 \%$ & 2 \\
\hline 61 a $80 \%$ & 8 \\
\hline 81 a $100 \%$ & 90 \\
\hline \multicolumn{2}{|l|}{ Fonte de renda } \\
\hline Agropecuária & 3 \\
\hline Pesca & 100 \\
\hline Comércio & 2 \\
\hline Transferência governamental (aposentadoria, bolsa família) & 6 \\
\hline Outros & 5 \\
\hline \multicolumn{2}{|l|}{ Renda mensal } \\
\hline Até $\mathrm{R} \$ 440,00$ & 2 \\
\hline De $\mathrm{R} \$ 441,00$ a $\mathrm{R} \$ 880,00$ & 50 \\
\hline De R\$ 881,00 a R \$ 1.320,00 & 41 \\
\hline De $R \$ 1.321,00$ a $R \$ 1.760,00$ & 7 \\
\hline Acima de $\mathrm{R} \$ 1.760,00$ & 0 \\
\hline
\end{tabular}

Fonte: Elaborado pelos autores.

Tabela 3. Estatística descritiva dos aspectos produtivos da pesca continental do município de Pentecoste, em 2016

\begin{tabular}{lcccc}
\hline \multicolumn{1}{c}{ Indicadores } & Mín. & Máx. & Média & DP $^{\mathbf{1}}$ \\
\hline Preço médio por quilo de peixe $(\mathrm{R} \$ / \mathrm{kg})$ & 2 & 7 & 3,28 & 1,286 \\
Quilos de peixe obtido por dia $(\mathrm{kg})$ & 8 & 30 & 13,93 & 5,143 \\
Tempo de pesca (h/dia) & 3 & 15 & 9,61 & 2,846 \\
\hline
\end{tabular}

Nota: (1) DP = Desvio padrão.

Fonte: Elaborado pelos autores.

cador capturava aproximadamente $14 \mathrm{~kg} /$ dia de peixe, mas em alguns casos a captura chegava ao mínimo de 8 $\mathrm{kg} /$ dia e até o máximo de $30 \mathrm{~kg} /$ dia. A média do tempo de pescaria foi de 10 horas/dia e alguns chegavam a pescar durante 15 horas.

A Tabela 4 apresenta as variáveis categóricas que foram utilizadas para caracterizar a pesca em Pentecoste.

O número de pescadores cadastrados na colônia de pescadores (Colônia Z16) foi de $64 \%$ da amostra. Estar cadastrado na colônia de pescadores e adim- plente com sua mensalidade garante ao pescador os benefícios do seguro defeso e da aposentadoria. O seguro defeso é concedido ao pescador profissional que exerce a atividade pesqueira de forma artesanal no valor de um salário mínimo vigente. ${ }^{7}$

7. O Instituto Brasileiro do Meio Ambiente e dos Recursos Naturais (Ibama) fixa anualmente o valo do seguro defeso, sendo este pago com recursos do Fundo de Amparo ao Trabalhador (FAT), instituído pela Lei no 7.998 de 11 de janeiro de 1990 (BRASIL, 2003). 
Tabela 4. Caracterização da pesca no município de Pentecoste, em 2016

\begin{tabular}{|c|c|c|c|}
\hline Indicadores & Percentual (\%) & Indicadores & Percentual (\%) \\
\hline Cadastrado na Colônica Z16 & 64 & Açude/Município & \\
\hline Possui embarcação & 85 & Pereira de Miranda (Pentecoste) & 100 \\
\hline Frequência de pesca & & Orós (Orós) & 46 \\
\hline Até 3 vezes por semana & 11 & Castanhão (Alto Santo) & 4 \\
\hline De 4 a 5 vezes por semana & 36 & Caxitoré (Umirim) & 57 \\
\hline De 6 a 7 vezes por semana & 53 & Boqueirão (Acarape) & 2 \\
\hline Espécie de peixe capturada & & Sítios novos (Caucaia) & 11 \\
\hline Tilápia do Nilo & 98 & Serrota (Apuiarés) & 21 \\
\hline Tucunaré & 53 & Maranguapinho (Maranguape) & 1 \\
\hline Curimatã & 80 & Consumo do pescado & \\
\hline Piau & 56 & $0 \%$ & 19 \\
\hline Traíra & 52 & $1-10 \%$ & 63 \\
\hline Pescada & 67 & $11-20 \%$ & 15 \\
\hline Camarão & 16 & $21-50 \%$ & 2 \\
\hline Beru & 33 & Mais de $50 \%$ & 1 \\
\hline Branquinha & 45 & Locais de venda & \\
\hline Sardinha & 3 & A intermediários & 88 \\
\hline \multirow{3}{*}{$\begin{array}{l}\text { Tambaqui, Pacu, Pirarucu, Carpa, } \\
\text { Apaiari, Pirambeba, Piranha }\end{array}$} & 0 & De casa em casa & 4 \\
\hline & & No mercado/feira & 22 \\
\hline & & Outra localidade/município & 0 \\
\hline
\end{tabular}

Fonte: Elaborado pelos autores.

Do total da amostra, 85\% dos pescadores têm embarcação. O pescador que não possui embarcação comumente pesca em regime de arrendamento ou "meia" da quantidade de peixe capturada. A embarcação utilizada na região era do tipo canoa a remo, de pequeno porte, que comporta apenas dois pescadores. $\mathrm{O}$ apetrecho de pesca comumente utilizado para capturar os peixes era a rede de espera. Quanto à frequência, a maioria dos pescadores (53\%) pescava quase que diariamente, ou seja, de seis a sete dias/semana.

A espécie capturada com maior frequência pelos pescadores foi a tilápia do Nilo, indicada por $98 \%$ dos pescadores. Em seguida, aparecem a curimatã $(80 \%)$, pescada $(67 \%)$, piau $(56 \%)$, tucunaré $(53 \%)$ e traíra $(52 \%)$. As espécies que receberam indicações inferiores a 50\% foram camarão, beru, branquinha, e sardinha. Algumas espécies foram apresentadas aos pescadores, mas não receberam indicações, como é o caso do tambaqui, pacu, pirarucu, carpa, apaiari, pirambeba e piranha.

Ao todo, foram indicados oito destinos de pescaria. Os pescadores da amostra pescavam com maior frequência nos açudes de Pereira de Miranda (100\%), Caxitoré (57\%) e Orós (46\%). Os açudes que receberam os menores percentuais de indicação foram Serrota
(21\%), Sítios Novos (11\%), Castanhão (4\%), Boqueirão $(2 \%)$ e Maranguape (1\%). Portanto, o pescador de Pentecoste tem ampliado suas oportunidades de pescaria em açudes localizados em vários municípios, além daquele de sua residência.

O produto da pescaria é destinado em sua maior parte à venda, como fica evidente ao observar o percentual de consumo do peixe capturado. Do total da amostra, $83 \%$ dos pescadores consumiram $10 \%$ ou menos do total capturado. A venda do peixe é feita, com maior frequência, ao intermediário ou no mercado/feira livre na sede do município. Segundo os respondentes, é frequente a venda do pescado ao intermediário já no próprio açude a preço abaixo do valor de mercado, sento tal prática motivada quase sempre pelo elevado grau de endividamento do pescador.

\subsection{Análise das preferências dos pescadores}

A análise da preferência dos pescadores inicia com a seleção das variáveis de interação a serem incluídas nos modelos logit, condicional e misto. Depois de testar a significância de vários termos de interação foram mantidas nos modelos as seguintes variáveis: 'pescar tilápia' e 'frequência de pesca de quatro dias ou mais 
por semana' (ETIL.MFREQP); 'pescar tilápia' e 'escolaridade maior ou igual a Ensino Fundamental incompleto' (ETIL.MESCOL); 'pescar peixes amazônicos' e 'maior faixa de renda proveniente da pesca', que corresponde a uma renda maior do que R $\$ 1.321$ (EPXAM. MREND); e 'custo de oportunidade de R\$ 41,60/dia' e 'estar cadastrado em alguma entidade' (COPORT. $C A D)$.

Em seguida, fez-se a comparação dos modelos logit, condicional e misto, quanto ao ajustamento dos dados. A Tabela 5 mostra os modelos logit, condicional e misto, estimados.

Usando o teste da razão de verossimilhança (LR) para comparar os modelos logit condicional, a versão expandida do modelo (MLC 2) foi a que melhor ajustou os dados de escolhas dos pescadores. Neste modelo, todas as variáveis explicativas apresentaram-se significantes ao nível de 5\%. Este resultado foi confirmado pelos outros critérios de ajustamento do modelo (pseudo $\mathrm{R}^{2}$ e os critérios de informação, AIC e BIC).
Com relação aos modelos logit mistos, os critérios de informação (AIC e BIC) não identificaram o melhor modelo, uma vez que os testes mostraram resultados contraditórios. Já a comparação de todos os modelos estimados mostrou que os modelos logit mistos tiveram melhor desempenho que os modelos logit condicionais.

Além disso, o teste de Hausman-McFadden mostrou que os dois modelos logit condicionais (MLC 1 e MLC 2) violaram o pressuposto IIA. Assim sendo, os coeficientes das variáveis são analisados tomando como base os modelos logit mistos, haja visto que esses modelos dispensam a observância do pressuposto de IIA e permitem controlar a heterogeneidade das preferências dos pescadores.

Nos modelos logit mistos (MLM 1), todos os coeficientes dos atributos se mostraram significativos ao nível de 5\% e apresentaram os sinais intuitivamente esperados, tendo tais resultados sido confirmados pelo MLM 2. Nesse modelo, as variáveis que tiveram coeficientes com sinais positivos foram: 'a quantidade

Tabela 5. Modelos logísticos que representam a pesca continental, em 2016

\begin{tabular}{|c|c|c|c|c|c|c|c|c|}
\hline \multirow{3}{*}{ Variáveis } & \multicolumn{4}{|c|}{ Modelo Logit Condicional } & \multicolumn{4}{|c|}{ Modelo Logit Misto } \\
\hline & \multicolumn{2}{|c|}{ MLC 1} & \multicolumn{2}{|c|}{$M L C 2$} & \multicolumn{2}{|c|}{ MLM 1} & \multicolumn{2}{|c|}{$M L M 2$} \\
\hline & Coefic. & $P>z$ & Coefic. & $P>z$ & Coefic. & $P>z$ & Coefic. & $P>z$ \\
\hline CAPTURA & 0,062 & 0,001 & 0,075 & 0,001 & 0,184 & 0,001 & 0,177 & 0,001 \\
\hline TILÁPIA & 0,820 & 0,001 & 2,007 & 0,001 & 1,117 & 0,001 & 2,532 & 0,001 \\
\hline PXAMA & $-0,634$ & 0,001 & $-0,744$ & 0,001 & $-0,682$ & 0,001 & $-0,799$ & 0,001 \\
\hline COMPX & 1,723 & 0,001 & 1,777 & 0,001 & 2,294 & 0,001 & 2,254 & 0,001 \\
\hline COPORT & $-0,007$ & 0,229 & $-0,033$ & 0,001 & $-0,018$ & 0,030 & $-0,045$ & 0,001 \\
\hline ETIL.MFREQP & & & $-1,087$ & 0,010 & & & $-1,342$ & 0,026 \\
\hline ETIL.MESCOL & & & $-0,624$ & 0,023 & & & $-0,781$ & 0,043 \\
\hline EPXAM.MREND & & & 1,192 & 0,022 & & & 1,249 & 0,039 \\
\hline COPORT.CAD & & & 0,034 & 0,001 & & & 0,040 & 0,003 \\
\hline DP (CAPTURA) & & & & & 0,157 & 0,001 & 0,129 & 0,001 \\
\hline DP (TILÁPIA) & & & & & 0,959 & 0,004 & 0,819 & 0,013 \\
\hline$D P(P X A M A)$ & & & & & 0,047 & 0,873 & 0,023 & 0,935 \\
\hline$D P(C O M P X)$ & & & & & 1,689 & 0,001 & 1,511 & 0,001 \\
\hline ASC & 0,515 & 0,113 & 0,603 & 0,071 & 1,364 & 0,002 & 1,390 & 0,002 \\
\hline № de obs. & \multicolumn{2}{|c|}{1800} & \multicolumn{2}{|c|}{1800} & \multicolumn{2}{|c|}{1800} & \multicolumn{2}{|c|}{1800} \\
\hline № de parâmetros & \multicolumn{2}{|c|}{6} & \multicolumn{2}{|c|}{9} & \multicolumn{2}{|c|}{6} & \multicolumn{2}{|c|}{9} \\
\hline Lrchi2 & \multicolumn{2}{|c|}{387,940} & \multicolumn{2}{|c|}{418,730} & \multicolumn{2}{|c|}{40,480} & \multicolumn{2}{|c|}{32,000} \\
\hline Pseudo $\mathrm{R}^{2}$ & \multicolumn{2}{|c|}{0,294} & \multicolumn{2}{|c|}{0,318} & & & & \\
\hline AIC & \multicolumn{2}{|c|}{942,395} & \multicolumn{2}{|c|}{919,604} & \multicolumn{2}{|c|}{909,916} & \multicolumn{2}{|c|}{895,603} \\
\hline BIC & & & & & \multicolumn{2}{|c|}{964,871} & \multicolumn{2}{|c|}{972,541} \\
\hline \multicolumn{9}{|c|}{ Teste da razão de verossimilhança para o MLC } \\
\hline LRchi2 (Lr/Lu) & \multicolumn{4}{|c|}{$\begin{array}{l}\text { Resultado do teste } \\
30,79\end{array}$} & \multicolumn{4}{|c|}{$\begin{array}{l}P>z \\
0,001\end{array}$} \\
\hline
\end{tabular}

Fonte: Elaborado pelos autores. 
capturada de quilos de peixe por dia' (CAPTURA), 'pescar tilápia' (TILÁPIA), 'açudes com peixamentos frequentes' (COMPX), 'custo de oportunidade' (COPORT) e a variável ASQ que identifica as escolhas de cenários alternativos que não o status quo.

Esses resultados estão de acordo com a expectativa do pescador em obter rendimentos crescentes da pescaria a partir de uma espécie de peixe de grande aceitação no mercado (tilápia do Nilo), estando esta produtividade associada à estocagem de peixes em açudes onde pesca. Arlinghaus et al. (2014), estudando a pesca continental na Alemanha, também observou que o pescador obtém maior utilidade ao escolher cenários que incluem uma determinada espécie de peixe.

As variáveis com coeficientes com valor negativo foram duas, 'pescar peixes amazônicos' (PXAMA) e 'custo de oportunidade de $\mathrm{R} \$ 41,60 /$ dia' (COPORT). Portanto, mantendo as outras variáveis constantes, quanto maior o custo de oportunidade e maior a captura de peixe amazônico, menor a utilidade esperada dos pescadores. Um aumento no custo de oportunidade da pescaria reduz a utilidade do pescador porque o custo do peixe capturado aumenta e a oportunidade de obter de renda em outra atividade é perdida. Este resultado está de acordo com a racionalidade econômica e confirma resultados obtidos em outros trabalhos (BLAMEY et al., 1999; ARAÚJO e COUTINHO, 2016).

No MLM 2, três termos de interação apresentaram coeficientes significativos ao nível de 5\%: EPXAM. MREND (Coef. = 1,249), ETIL.MESCOL $(-0,781)$, COPORT.CAD (Coef. $=0,040$ ) e ETIL.MFREQP (Coef. $=-1,342)$. Com base no sinal dos coeficientes dessas variáveis, observa-se que: 'pescar peixe amazônico' e 'ter renda mensal acima de $\mathrm{R} \$ 1.321$ ' aumentaram a utilidade do pescador; 'pescar tilápia' e 'ter nível médio de escolaridade' declinaram a utilidade do pescador; 'custo de oportunidade de R\$ 41,60/dia' e 'ser cadastrado na colônia' aumentaram a utilidade do pescador; e 'pescar tilápia' e 'ter frequência de pescaria de quatro dias ou mais' também diminuíram a utilidade do pescador.

A interpretação das relações de causalidade implícitas nos termos de interação não é clara; porém, tais relações são úteis para estabelecer hipóteses sobre a preferência do pescador que podem ser investigadas em estudos futuras.

Do ponto de vista das políticas públicas, conhecer a relação entre o efeito marginal de uma característica da pescaria sobre a utilidade do pescador é importante, em geral, para orientar o planejamento e gerenciamento dos açudes públicos e, particularmente, formatar o programa de peixamento.

Neste sentido, as preferências do pescador por uma pescaria eficiente apontaram a necessidade de estocar frequentemente os açudes, introduzir espécies de peixe com valor comercial (e.g. tilápida do Nilo) e aumentar a quantidade individual de peixe capturado por dia. Ficou evidente também que a situação corrente (status quo) não está gerando a utilidade esperada pelo pescador e que ganhos de utilidade podem ser obtidos empreendendo mudanças nas características dos açudes onde se realiza a pescaria.

\subsection{Disposição a pagar dos pescadores}

As médias das disposições a pagar (DAP) foram estimadas com base no modelo logit misto expandido (MLM 2). A Tabela 6 apresenta as estimativas da disposição a pagar do pescador por mudança nos atributos da pesca continental.

As disposições médias a pagar foram positivas para 'quantidade capturada de quilos de peixe por dia', 'pescar tilápia' e 'peixamento do açude'. A disposição a pagar foi negativa apenas para 'pescar peixes amazônicos'. O valor negativo da DAP tem sido interpretado como uma disposição a receber (DAR) pelo bem ou

Tabela 6. Disposição a pagar por mudança nos atributos da pesca continental no MLM 2, em 2016

\begin{tabular}{lcccc}
\hline \multicolumn{1}{c}{ Variáveis } & Unid. & DAP Média & Desvio Padrão & $\mathbf{P}>\mathbf{Z}$ \\
\hline ASC & R\$/dia & 30,656 & 14,265 & 0,032 \\
CAPTURA & R\$/kg.dia & 3,896 & 1,329 & 0,003 \\
TILÁPIA & R\$/dia & 5,585 & 19,997 & 0,005 \\
PXAMA & R\$/dia & $-17,627$ & 6,555 & 0,007 \\
COMPX & R\$/dia & 49,723 & 15,224 & 0,001 \\
\hline
\end{tabular}

Fonte: Elaborado pelos autores. 
serviço ambiental pelo fato de este atributo causar uma diminuição do nível de utilidade. Portanto, o pescador deveria receber uma compensação monetária por pescar peixes amazônicos no valor de $\mathrm{R} \$ 17,6 /$ dia.

As DAPs médias estimadas apresentaram-se estatisticamente significativas a um nível de 5\% de significância. A variável que apresentou a maior DAP média foi a de pescar em açudes onde são realizados peixamentos frequentemente (COMPX). O pescador está disposto a pagar $\mathrm{R} \$ 49,72 /$ dia a mais para pescar em açudes que foram peixados com frequência do que pagaria em açudes não peixados. Isto porque, quanto maior a frequência de peixamento, maior será a chance de o pescador aumentar a quantidade capturada e, consequentemente, a sua renda.

O segundo maior valor da disposição a pagar dos pescadores foi atribuído à variável ASC. Como já mencionado anteriormente, esta variável representa todas as escolhas de cenários feitas pelo pescador que não o próprio status quo (SQ). Portanto, o pescador estaria disposto a pagar, em média, $\mathrm{R} \$ 30,70 /$ dia por qualquer cenário que não seja o status quo (SQ).

$\mathrm{O}$ pescador apresentou a menor disposição a pagar média para o aumento da quantidade de peixe capturada diariamente (CAPTURA), que foi de aproximadamente $R \$ 4,00 / \mathrm{kg}$.dia. Esse valor mostrou-se relativamente baixo talvez devido à menor amplitude de variação na quantidade capturada diária proposta nos cenários, tendo tal amplitude refletida na situação corrente de seca na região.

A Tabela 7 apresenta as probabilidades de escolhas estimadas pelo modelo logit misto expandido para os cenários propostos, o ranking das escolhas e a estatística descritiva da disposição a pagar por cada cenário.

A disposição a pagar média por cenário e sua probabilidade de escolha mostraram-se consistentes, ou seja, cenários com maior disposição a pagar média (DAP) apresentaram maior probabilidade de escolha e vice-versa.

Os dois cenários que apresentaram as maiores probabilidades de escolha e as maiores disposições a pagar médias foram o C3 $(0,821)$ e $C 4(0,669)$. Esses cenários são formados pelos níveis dos atributos que proporcionam ganhos de utilidade para os pescadores, que é pescar tilápia, ter acesso a açudes que são peixados com frequência, dedicar o tempo mínimo ou médio de tempo na pescaria ( 2 e 4 horas/dia) e pescar uma quantidade próxima da média de captura (5 a $8 \mathrm{~kg} / \mathrm{dia}$ ).

Já os três cenários com as menores probabilidades de escolha foram C2 $(0,231)$, SQ $(0,141)$ e C8 $(0,129)$. Os pescadores estavam dispostos a pagar R $\$ 19,61 /$ dia para experimentar $C 2$ e receber compensação de $R \$$ 14,117 pelo cenário C8. A baixa preferência do pescador por esses cenários pode ser explicada pelos níveis dos atributos que compõem esses cenários. Por exemplo, o cenário $C 8$ consiste da captura de $5 \mathrm{~kg}$ de peixes amazônicos durante duas horas em açude que não é peixado com frequência.

Finalmente, a DAP média global para a escolha de qualquer um dos cenários propostos foi estimada em R\$ 89,62/dia, que variou no intervalo entre o valor mínimo de -R \$14,12/dia e o máximo de $\mathrm{R} \$ 151,78 /$ dia $(\mathrm{DP}=\mathrm{R} \$ 45,00 /$ dia $)$. Esta DAP média mostra que ganhos de bem-estar para o pescador de Pentecoste

Tabela 7. Probabilidade, ranking das escolhas e a estatística descritiva da DAP média dos pescadores, em 2016

\begin{tabular}{|c|c|c|c|c|c|c|}
\hline Cenários & Probabilidade & Ranking & $\begin{array}{c}\text { DAP Média (R\$/ } \\
\text { dia) }\end{array}$ & $\begin{array}{l}\text { Desvio padrão } \\
\text { (R\$/dia) }\end{array}$ & $\begin{array}{l}\text { Mín. } \\
\text { (R\$/dia) }\end{array}$ & $\begin{array}{c}\text { Máx. } \\
\text { (R\$/dia) }\end{array}$ \\
\hline$S Q$ & 0,141 & 90 & - & - & - & - \\
\hline $\mathrm{C} 1$ & 0,472 & $3^{\mathrm{o}}$ & 81,073 & 0,000 & 81,073 & 81,073 \\
\hline$C 2$ & 0,231 & $8 \mathrm{o}$ & 19,610 & 14,483 & 17,049 & 98,978 \\
\hline C3 & 0,821 & $1^{o}$ & 151,607 & 1,823 & 132,061 & 151,776 \\
\hline C4 & 0,669 & $2^{\mathrm{o}}$ & 118,458 & 6,699 & 54,906 & 119,156 \\
\hline C5 & 0,470 & $4^{\mathrm{o}}$ & 79,026 & 0,000 & 79,026 & 79,026 \\
\hline C6 & 0,299 & $7^{\circ}$ & 42,619 & 5,135 & 41,764 & 72,573 \\
\hline C7 & 0,449 & $5^{\mathbf{o}}$ & 73,811 & 9,900 & 72,573 & 151,776 \\
\hline C8 & 0,129 & $10^{\mathrm{o}}$ & $-14,117$ & 0,000 & $-14,117$ & $-14,117$ \\
\hline C9 & 0,300 & $6^{\mathrm{o}}$ & 30,538 & 0,000 & 30,538 & 30,538 \\
\hline Média Global & - & - & 89,623 & 44,942 & $-14,117$ & 151,776 \\
\hline
\end{tabular}

Fonte: Elaborado pelos autores. 
podem ser alcançados por meio da melhoria nos níveis dos atributos que caracterizam a experiência da pescaria na região.

Do ponto de vista da gestão dos açudes, as políticas que buscam a eficiência econômica da pesca continental nos açudes do semiárido deveriam buscar reproduzir nos açudes os níveis dos atributos daqueles cenários que apresentaram maior probabilidade de escolha.

\section{Considerações finais}

Dentre os modelos testados, o modelo logit misto expandido ajustou melhor os dados das escolhas. Neste modelo, todos os coeficientes se mostraram significativos ao nível de 5\% e com os sinais esperados. Quando comparado aos atributos na situação corrente (status quo), os níveis dos atributos que contribuíram para aumentar a utilidade do pescador foram quantidade capturada em quilos por dia, pescar tilápia e pescar em açudes que sejam peixados frequentemente. Os atributos que declinaram a utilidade do pescador foram o custo de oportunidade de $\mathrm{R} \$ 41,60 /$ dia e pescar peixes amazônicos.

As estimativas das disposições a pagar marginais (DAPs) dos níveis dos atributos mostraram-se consistentes apresentando uma relação direta com os coeficientes gerados pelo modelo logit misto expandido. Os pescadores estavam dispostos a pagar valores positivos pelo aumento na quantidade capturada de quilos peixe por dia ( $\mathrm{R} \$ 3,896 /$ dia), pescar tilápia (R\$ 5,585/dia), pescar em açudes que são frequentemente peixados (R\$ 49,723) e trocar a situação atual por qualquer outro cenário proposto (R\$ 30,656/dia). Contrariamente, estavam dispostos a receber compensação pela captura de peixes amazônicos ( $\mathrm{R} \$ 17,627$ ) dia). Os pescadores atribuíram maior peso aos ganhos de utilidade proporcionados por pescar em açudes que são peixados frequentemente.

Os cenários que apresentaram maior probabilidade de escolha pelos pescadores, em trocar a situação atual $(S Q)$ pelo cenário apresentado, continham níveis preferidos pelos pescadores, sendo a maior DAP média de aproximadamente $\mathrm{R} \$ 152,00 /$ dia.

Em média, a disposição a pagar marginal do pescador pela mudança nos atributos da pesca atual foi estimada em R\$ 89,00/dia. Esse resultado evidenciou que o pescador continental do semiárido do Nordeste, que reside no município de Pentecoste, tem ganhos de benefícios pela substituição dos atributos da pesca corrente por aqueles atributos que refletem a sua preferência.

\section{Referências}

ARAÚJO, R. C. P. e COUTINHO, A. C. Disposição a pagar por métodos de cobrança da água bruta no perímetro irrigado dos tabuleiros de Russas, Ceará-Br. Revista de Gestão de Água da América Latina, v. 13, n. 1, jan./jun. 2016.

ARLINGHAUS, R. et al. Species-specific preferences of German recreational anglers for freshwater fishing experiences, with emphasis on the intrinsic utilities of fish stocking and wild fishes. Journal of Fish Biology, Spain, v. 85, n. 6, p. 1843-1867, 2014.

BARTON, D. N. e BERGLAND, O. Valuing irrigation water using a choice experiment: an 'individual status quo' modeling of farm specific water scarcity. Environment and Development Economics, Rio de Janeiro, v. 15, n. 3, p. 321-340, 2010.

BATEMAN, J. et al. Economic valuation with stated preference techniques: a manual. Cheltenham-UK: Edward Elgar, 2002.

BELL, A. R., SHAH, M. A. A. e WARD, P. S. Reimagining cost recovery in Pakistan's irrigation system through willingness-to-pay estimates for irrigation water from a discrete choice experiment. Water Resources Research, Spain, v. 50, p. 6679-6695, 2014.

BLAMEY, R., GORDON, J. e CHAPMAN, R. Choice modeling: assessing the environmental values of water supply options. The Australian Journal of Agricultural and Resource Economics, Spain, v. 43, n. 3, p. 337-357, 1999.

BRASIL. Lei no 10.779, de 25 de novembro de 2003. Dispõe sobre a concessão do benefício de seguro desemprego, durante o período de defeso, ao pescador profissional que exerce a atividade pesqueira de forma artesanal. DOU. Brasília, DF, 25 nov. 2003. Disponível em: <http://www.planalto.gov.br/ccivil_03/leis/2003/ L10.779.htm >. Acesso em: 13 fev. 2017.

Ministério da Transparência, Fiscalização e Controladoria-Geral da União. Despesas com o pescador artesanal no município de Pentecoste no ano de 2015. Portal da Transparência, 2015. Disponível em: $<$ http://www.portaltransparencia.gov.br/defeso/ defesoListaMunicipios.asp $? \mathrm{UF}=\mathrm{CE} \&$ Pagina $=1 \&$ Texto Pesquisa $=$ pentecoste $>$. Acesso em: 08 fev. 2017. 
BUCHANAN, J. M. Opportunity cost. The New Palgrave Dictionary of Economics, Madison, v. 3, p. 718, 1987.

CEARÁ. Secretaria do Planejamento e Gestão. Instituto de Pesquisa e Estratégia Econômica do Ceará, IPECE. Perfil básico municipal 2015 de Pentecoste. Fortaleza, 2015. Disponível em: < http://www.ipece.ce.gov.br/perfil_ basico_municipal/2015/ Pentecoste.pdf $>$. Acesso em: 24 jun. 2016.

COUTINHO, A. C. Análise das preferências dos irrigantes por sistema de cobrança pelo uso da água bruta: o caso do perímetro irrigado de tabuleiro de russas. 2015. $167 \mathrm{f}$. Dissertação (Mestrado em Desenvolvimento e Meio Ambiente) - Universidade Federal do Ceará Fortaleza, 2015.

COGERH - Companhia de Gerenciamento dos Recursos Hídricos. Portal Hidrológico do Ceará. Disponível em: <http://www.hidro.ce.gov.br/>. Acesso em: 24 jun. 2016.

DITTON, R. B. Understanding angler preferences for harvest regulations by level of species preference. Northeastern Recreation Research Symposium, Austin, 2004, p. 185-181.

IBGE. Censo Demográfico 2010. Brasília, 2010. Disponível em: <www.ibge.gov.br>. Acesso em: 14 jun. 2016.

MCFADDEN, D. e TRAIN, K. Mixed MNL models for discrete response. Journal Applied Econometrics, Spain, v. 15, p. $447-470,2000$.

PEARCE, D. (Ed.). Environmental valuation in developed countries: case studies. Edward Elgar, Cheltenham, UK, 2006.
RIGBY, D., ALCON, F. e BURTON, M. Supply uncertainty and the economic value of irrigation water. European Review of Agricultural Economics, Oxford, v. 37, n. 1, 2010.

RODRIGUES, B. F. Valoração econômica dos recursos pesqueiros na região do lago de Manacapuru. Dissertação (Mestrado em Ciências do Ambiente e Sustentabilidade da Amazônia) - Centro de Ciências do Ambiente, Universidade Federal do Amazonas, Manaus, 2010.

SALES, L. T. Avaliação dos peixamentos realizados em açudes das bacias dos rios Brígida, Terra Nova, Pajeú e Moxotó (Pernambuco - Brasil). Dissertação (Mestrado em Gestão e Políticas Ambientais) - Universidade Federal de Pernambuco, Recife, p. 108, 2001.

SALES, A. M. P. Peixamentos realizados pelo centro de pesquisas em aquicultura Rodolpho Von Ihering. Monografia (Graduação) - Curso de Engenharia de Pesca, Departamento de Engenharia de Pesca, Universidade Federal do Ceará - UFC, Fortaleza, p. 35, 2015.

SILVA, J. W. B. Tilápias: biologia e cultivo. Evolução, situação atual e perspectivas da tilapicultura no Nordeste Brasileiro. Fortaleza: Edições UFC, 2009.

SILVA, S. M. O. Sustentabilidade do programa de peixamento do governo do estado do Ceará em coleções de águas públicas. Dissertação (Mestrado em Economia Rural) - Departamento de Economia Agrícola da Universidade Federal do Ceará, Fortaleza, p. 86, 2011.

SILVA, S. M. O. e PINHEIRO, J. C. V. Índice de sustentabilidade do Programa de Peixamento no Município de Canindé-CE. Revista Econômica do Nordeste, Fortaleza, v. 44, n. 1, p. 163-181, 2013.

Todo o conteúdo deste periódico, exceto onde estiver identificado, está licenciado sob uma Licença Creative Commons (cc by 4.0). 
